\title{
PREDIÇÃO DO CICLO DE CORTE DE ESPÉCIES ARBÓREAS COMERCIAIS POR GRUPOS ECOLÓGICOS EM UMA FLORESTA NA AMAZÔNIA BRASILEIRA
}

\author{
Larissa Corrêa Lopes Quadros OLIVEIRA ${ }^{1}$ \\ Fernando Cristovam da Silva JARDIM ${ }^{2}$ \\ Jaqueline Macedo GOMES ${ }^{3}$
}

- RESUMO: Pesquisas são necessárias para subsidiar os silvicultores à manejarem a floresta visando melhor aproveitamento. Para auxiliar as pesquisas pode-se, analisando a distribuição diamétrica, agrupar as espécies de acordo com as suas características em relação ao crescimento diamétrico e projetar o seu crescimento futuro, determinando se atingiu o diâmetro esperado antes do ciclo de corte estipulado em lei. O trabalho teve como objetivo realizar a prognose da distribuição diamétrica da floresta utilizando o modelo Matriz de Transição para as espécies classificadas como tolerantes, intolerantes e intermediárias e definir o ciclo de corte por grupos ecológicos, a fim de verificar se o tempo definido na legislação ambiental brasileira é compatível com a recuperação e crescimento das espécies arbóreas. A área da pesquisa localiza-se em Moju, Pará-Brasil. Selecionou-se 9 clareiras, em torno das quais instalou-se 4 parcelas amostrais de 500 $\mathrm{m}^{2}$. Foram medidos todos os indivíduos com DAP $\geq 5 \mathrm{~cm}$. Para a prognose, utilizou-se os dados reais dos anos 2007 e 2010 e fez-se as projeções para períodos de 3 anos (2013 a 2028). Com os dados prognosticados fez-se a estimativa do volume até 31 anos após exploração florestal. Constatou-se que para as espécies tolerantes e intolerantes o ciclo de corte ideal é de 16 anos e para as intermediárias é de 22 anos.

- PALAVRAS-CHAVE: Exploração florestal; manejo florestal; matriz de transição; distribuição diamétrica.

\section{Introdução}

A Instrução Normativa ${ }^{\circ} 05$ de 2006 do Ministério do Meio Ambiente (Brasil, 2006) define um ciclo de corte inicial de 25 a 35 anos para plano de Manejo Florestal Sustentável pleno que prevê a utilização de máquinas para o arraste de toras. Estabelece também que a intensidade máxima de corte a serem autorizadas é de $30 \mathrm{~m}^{3} \mathrm{ha}^{-1}$, com ciclo de corte de 35 anos. Em seu artigo $9^{\circ}$, afirma que somente poderá ser requerida a redução do ciclo de corte quando comprovada a recuperação da floresta.

\footnotetext{
${ }^{1}$ Universidade Federal Rural da Amazônia - UFRA, Instituto de Ciências Agrária, CEP: 66077-530, Belém, PA, Brasil, E-mail: larissaclquadros@gmail.com

${ }^{2}$ Universidade Federal Rural da Amazônia - UFRA, UFRA, Instituto de Ciências Agrária, CEP: 66077-530, Belém, PA, Brasil. (In memoriam)

${ }^{3}$ Universidade Estadual da Região Tocantina do Maranhão - UEMASUL, CEP: 65900-000, Imperatriz, MA, Brasil, E-mail: jaquelinemacedogomes@hotmail.com; jaquelinegomes@uemasul.edu.br
} 
A fixação de um ciclo de corte capaz de permitir o crescimento diamétrico das espécies de valor comercial é primordial, pois este parâmetro é um instrumento regulador da quantidade de madeira removida e está diretamente relacionado com o estoque de crescimento. No entanto, a complexidade dos ecossistemas de florestas tropicais dificulta a avaliação do crescimento e da produção florestal e consequentemente a definição de um ciclo de corte adequado para as espécies (SOUZA et al., 2004). O entendimento do comportamento das taxas de crescimento, recrutamento e mortalidade, são importantes para definir quanto tempo uma espécie, ou um grupo de espécies demora para atingir uma determinada dimensão (TEIXEIRA et al., 2007).

A disponibilidade de informações sobre o padrão de crescimento das árvores individuais é um trunfo que permite a seleção de espécies para a exploração ou proteção, bem como, a estimativa de ciclos de corte e prescrição de tratamentos silviculturais (SILVA et al., 2002).

A modelagem do crescimento e da produção visa contribuir com as atividades do manejo, uma vez que tem o poder de prever o momento em que a floresta recupera seu estoque após a exploração florestal. Segundo Nascimento et al. (2014), a tomada de decisão na gestão das florestas exige previsões precisas de crescimento e produção em diferentes cenários capazes de fornecer várias possibilidades para a exploração madeireira.

A prognose é aplicada com a finalidade de predizer a estrutura e a produção da floresta; avaliar a sustentabilidade ambiental e econômica do manejo florestal; definir a necessidade, época e intensidade de aplicação de tratamentos silviculturais; avaliar efeitos e alternativas de manejo da floresta; predizer o ciclo de corte e fazer estudos de cenários (Souza e Soares, 2013).

Diferentes metodologias para gerar projeções em florestas heterogêneas estão descritas na literatura, e, dentre essas, pode-se citar a matriz de transição que é um modelo de distribuição de diâmetro. Segundo Vanclay (1994) os modelos de distribuição de diâmetro são os mais difundidos para realizar a prognose, em decorrência da facilidade de obtenção de dados e têm sido testados para predizer a estrutura das florestas.

Para facilitar os estudos, considerando a diversidade da floresta Amazônica, pode-se agrupar as espécies arbóreas com características ecológicas e silviculturais semelhantes, como formas de vida, forma de crescimento, estratégias de vida, demanda por radiação solar, entre outras (BAKER, 1949; BUDOWSKI, 1965; DENSLOW, 1980; HARTSHORN, 1980; WHITMORE, 1984; CORNELISSEN et al., 2003; KAGEYAMA e GANDARA, 2004; HUMBERT et al., 2007; COELHO et al. 2012; DUAH-GYAMFI et al., 2014; SOARES et al., 2016). A classificação das espécies com propriedades parecidas facilita a proposição de metodologias aplicáveis a cada agrupamento.

De acordo com Vale et al. (2011), agrupar as espécies, quanto as suas características funcionais, é fundamental para se compreender as suas relações com o local onde elas se encontram distribuídas. Ferraz et al. (2004) afirmam que uma classificação facilitará modelar a dinâmica da floresta e o conhecimento pode ser aplicado diretamente na conservação e no manejo sustentado.

Neste estudo pretendeu-se responder a seguinte questão: o ciclo de corte definido na legislação ambiental brasileira é compatível com a recuperação e crescimento das espécies arbóreas? O objetivo foi realizar a prognose da distribuição diamétrica da floresta utilizando o modelo matriz de transição e definir o ciclo de corte por grupos ecológicos das espécies comerciais e de toda a floresta explorada. 


\section{Material e métodos}

\section{1 Área de estudo}

A área de estudo localiza-se no Campo Experimental da Embrapa Amazônia Oriental, $\mathrm{Km} 30$ da Rodovia PA 150, no município de Moju, Pará, situado entre as latitudes $2^{\circ} 07^{\prime} 30^{\prime \prime} \mathrm{S}$ e $2^{\circ} 12^{\prime} 06^{\prime}$ 'S e longitudes $48^{\circ} 46^{\prime} 57^{\prime \prime} \mathrm{W}$ e $48^{\circ} 48^{\prime} 30^{\prime \prime} \mathrm{W}$ do meridiano de Greenwich. A área possui 1.059 ha, na qual foram explorados seletivamente 200 ha.

O clima da região é do tipo Am (clima quente e úmido), segundo a classificação de Köppen, com temperaturas médias mensais oscilando entre $21^{\circ} \mathrm{C}$ e $33^{\circ} \mathrm{C}$ e a média anual é em torno de $26^{\circ} \mathrm{C}$. A precipitação pluviométrica anual varia de 2.000 a $3.000 \mathrm{~mm}$, distribuída irregularmente. A umidade relativa do ar está em torno de $85 \%$. (Silva et al., 2001).

O relevo é plano, com pequenas ondulações. Predomina na área o Latossolo Amarelo distrófico com diferentes texturas ocorrendo também solos Podzólicos VermelhosAmarelos, Glei Pouco Úmico e Plintossolo. A tipologia da área experimental é Floresta Ombrófila Densa de terra firme (Silva et al., 2001).

\subsection{Coleta de dados}

Dentro da Estação Experimental da Embrapa, foi selecionada uma área de 200 ha, a qual sofreu exploração florestal seletiva em 1997, através de uma parceria entre a Embrapa e a empresa madeireira Perachi. Antes da colheita foi realizado o corte de todos os cipós com diâmetro $\geq 2 \mathrm{~cm}$ nos 200 ha. Foram colhidas, em média, 3,3 árvores ha ${ }^{-1}$ com diâmetro mínimo de corte (DMC) de $65 \mathrm{~cm}$, de 24 espécies comerciais, correspondendo a um volume de $23 \mathrm{~m}^{3} \mathrm{ha}^{-1}$, que representou $69 \%$ do volume planejado de $33,5 \mathrm{~m}^{3} \mathrm{ha}^{-1}$ (SILVA et al., 2001; LOPES et al., 2001; REIS et al., 2017) (Tabela 1).

Nessa área foram selecionadas nove clareiras provenientes da exploração, em torno das quais foi realizada a marcação das parcelas amostrais do projeto "Avaliação da dinâmica florestal após exploração madeireira seletiva". Em cada uma das nove clareiras selecionadas foram instaladas faixas de $500 \mathrm{~m}^{2}$, começando na bordadura da clareira para dentro da floresta, nas direções norte, sul, leste e oeste, portanto, quatro faixas por clareira. Dentro dessas parcelas foram inventariadas árvores com DAP $\geq 5 \mathrm{~cm}$, consideradas da população adulta.

Os espécimes levantados foram identificados por nomes vulgares e tiveram material vegetativo coletado para a determinação dos nomes científicos a partir da comparação nos Herbários IAN da Embrapa Amazônia Oriental e do Museu Emílio Goeldi. O sistema de classificação botânica utilizado foi o de "Angiosperm Phylogeny Group" (APG III, 2009) e a nomenclatura botânica conferida com o banco de dados eletrônico disponibilizado pelo Programa REFLORA (2017).

Foram feitas 16 medições entre os anos 1998 e 2010. Para o estudo foram utilizadas as medições de 2007 e 2010. 
Tabela 1 - Espécies planejadas para colheita em 1997 em 200 ha de uma Floresta Tropical de Terra Firme, no município de Moju, PA

\begin{tabular}{|c|c|c|c|c|}
\hline Nome vulgar & Nome científico & $\mathbf{N}$ & N/ha & $\mathbf{m}^{3} / \mathbf{h a}$ \\
\hline Caju-açu & $\begin{array}{l}\text { Anacardium giganteum } \mathrm{W} . \\
\text { Hancock ex Engl. }\end{array}$ & 30 & 0,15 & 1,10 \\
\hline Mururé & $\begin{array}{c}\text { Brosimum acutifolium subsp. } \\
\text { Obovatum (Ducke) }\end{array}$ & 33 & 0,165 & 1,08 \\
\hline Piquiarana & Caryocar glabrum Pers. & 22 & 0,11 & 1,10 \\
\hline Piquiá* & Caryocar villosum (Aubl.) Pers.* & 10 & 0,05 & 0,42 \\
\hline Tauarí* & $\begin{array}{l}\text { Couratari oblongifolia Ducke \& } \\
\text { R. Knuth* }\end{array}$ & 42 & 0,21 & 1,83 \\
\hline $\begin{array}{l}\text { fava- } \\
\text { mapuxiqui* }\end{array}$ & Dimorphandra gardneriana Tul.* & 19 & 0,095 & 0,59 \\
\hline $\begin{array}{l}\text { angelim- } \\
\text { pedra* }\end{array}$ & Dinizia excelsa Ducke* & 16 & 0,08 & 1,12 \\
\hline Cupiúba & Goupia glabra Aubl & 73 & 0,365 & 3,13 \\
\hline $\begin{array}{l}\text { Angelim- } \\
\text { vermelho* }\end{array}$ & Hymenaea $\mathrm{sp}^{*}$ & 6 & 0,03 & 0,38 \\
\hline $\begin{array}{l}\text { Jutaí-açú / } \\
\text { Jatobá }\end{array}$ & Hymenaea courbaril L. & 15 & 0,075 & 0,69 \\
\hline $\begin{array}{l}\text { Angelim-da- } \\
\text { mata }\end{array}$ & Hymenolobium excelsum Ducke & 6 & 0,03 & 0,23 \\
\hline Maparajuba* & $\begin{array}{c}\text { Manilkara bidentata (A. DC.) A. } \\
\text { Chev.* } \\
\text { Manilkara paraensis (Huber) } \\
\text { Standl. }\end{array}$ & 10 & 0,05 & 0,34 \\
\hline
\end{tabular}

$\mathrm{N}$ : número de árvores planejadas para colheita; N/ha: número de árvores por hectare planejadas para colheita; $\mathrm{m}^{3} /$ ha: volume planejado para colheita por espécie; * espécies que não estão presentes nas parcelas amostrais. 
Tabela 1 (continuação) - Espécies planejadas para colheita em 1997 em 200 ha de uma Floresta Tropical de Terra Firme, no município de Moju, PA

\begin{tabular}{|c|c|c|c|c|}
\hline Nome vulgar & Nome científico & $\mathbf{N}$ & N/ha & $\mathbf{m}^{3} / \mathbf{h a}$ \\
\hline Maçaranduba & $\begin{array}{l}\text { Manilkara huberi (Ducke) A. } \\
\text { Chev. }\end{array}$ & 155 & 0,775 & 5,81 \\
\hline $\begin{array}{l}\text { louro- } \\
\text { vermelho }\end{array}$ & Ocotea rubra Mez & 21 & 0,105 & 0,86 \\
\hline $\begin{array}{c}\text { fava- } \\
\text { barriguga }\end{array}$ & Parkia gigantocarpa Ducke & 47 & 0,235 & 1,82 \\
\hline fava-bolota* & $\begin{array}{c}\text { Parkia pendula (Willd.) Benth. ex } \\
\text { Walp.* }\end{array}$ & 15 & 0,075 & 0,73 \\
\hline $\begin{array}{l}\text { angelim- } \\
\text { rajado }\end{array}$ & $\begin{array}{c}\text { Zigia racemosa (Ducke) Barneby } \\
\text { \& J.W. Grimes }\end{array}$ & 7 & 0,035 & 0,34 \\
\hline saboeiro & $\begin{array}{c}\text { Abarema jupumba (Willd.) Britton } \\
\text { \& Killip }\end{array}$ & 14 & 0,07 & 0,42 \\
\hline $\begin{array}{l}\text { faveira fl. } \\
\text { Fina* }\end{array}$ & $\begin{array}{c}\text { Pseudopiptadenia suaveolens } \\
\text { (Miq.) J.W. Grimes* }\end{array}$ & 106 & 0,53 & 3,62 \\
\hline $\begin{array}{l}\text { mandioqueira } \\
\text {-aspera }\end{array}$ & Qualea albiflora Warm. & 16 & 0,08 & 0,77 \\
\hline axixá & Sterculia excelsa Mart & 27 & 0,135 & 0,77 \\
\hline tanimbuca & $\begin{array}{l}\text { Terminalia amazonia (J.F.Gmel.) } \\
\text { Exell }\end{array}$ & 34 & 0,17 & 1,29 \\
\hline $\begin{array}{l}\text { breu- } \\
\text { sucuruba }\end{array}$ & Trattinnickia rhoifolia Willd. & 12 & 0,06 & 0,57 \\
\hline \multirow[t]{2}{*}{ acapu } & Vouacapoua americana Aubl. & 139 & 0,695 & 4,54 \\
\hline & TOTAL & 875 & 4,37 & 33,47 \\
\hline
\end{tabular}

N: número de árvores planejadas para colheita; N/ha: número de árvores por hectare planejadas para colheita; $\mathrm{m}^{3} /$ ha: volume planejado para colheita por espécie; * espécies que não estão presentes nas parcelas amostrais. (Fonte: Edital de licitação No 00/96 - Embrapa Amazônia Oriental e Autorização para exploração de Plano de Manejo Florestal Sustentável (PMFS) - No 45/96 - IBAMA, elaborado por Reis et al., 2017).

\subsection{Análise de dados}

Para avaliar se a floresta explorada recuperou o seu volume de madeira e verificar se o ciclo de corte estabelecido na legislação ambiental brasileira é compatível com a recuperação e crescimento das espécies arbóreas, fez-se a prognose da distribuição diamétrica das espécies exploradas, partindo dos anos 2007-2010 e projetando para os anos 2013, 2016, 2019, 2022, 2025 e 2028, até 31 anos após a exploração, utilizando o modelo matriz de transição.

A distribuição diamétrica das espécies arbóreas foi analisada a partir do DAP $\geq 5 \mathrm{~cm}$ em intervalos com amplitude de $10 \mathrm{~cm}$ de diâmetro, conforme Instrução Normativa brasileira $n^{\circ} 05$ de 2006 do Ministério do Meio Ambiente. 
A matriz de transição é um instrumento que o manejador pode recorrer para prever o recrutamento, a mortalidade e o estoque por classe diamétrica de uma floresta ineqüiânea ao longo do tempo (SANQUETTA et al., 1996). Sua representação matemática pode ser escrita como na equação 1:

$$
A \times E 0+R=E 1,
$$

em que: $A=$ matriz de transição que contém probabilidades de uma árvore passar para outras classes diamétricas através de crescimento com o tempo; $E 0=$ vetor de estoque no momento zero, ou seja, a distribuição diamétrica antes da predição; $R=$ vetor de recrutamento; $E 1$ = vetor de estoque no momento um, no tempo de predição futuro (SANQUETTA et al., 1996).

A Matriz de Transição foi estruturada em 9 estados: $e_{I}$ (recrutamento $\left.=\mathrm{R}\right), e_{2}$ a $e_{8}$ (classes diamétricas com amplitude de $10 \mathrm{~cm}), e_{09}($ mortalidade $=\mathrm{M})$. O recrutamento é a mudança do estado $e_{1}$ para qualquer dos estados $e_{2}$ a $e_{8}$; a classe diamétrica é a mudança para as classes diamétricas superiores por meio do incremento diamétrico, ou permanência na mesma classe quando o incremento não é suficiente para mudança de classe; e a mortalidade é a mudança de qualquer estado para o estado $e_{0}$. Após a definição dos estados foi montada uma tabela com o número de indivíduos em cada estado e posteriormente calculada a matriz de transição probabilística (pi,j) (Equação 2).

$$
p i, j=\frac{n i j}{N j}
$$

em que: $p i, j=$ a probabilidade de ocorrência de indivíduos na classe $\mathrm{j}$ no tempo $t+1$ (segunda ocasião considerada), dado a classe $i$ no tempo $t$ (primeira ocasião considerada); $n i j=0$ número de indivíduos na classe $j$ no tempo $t+1$ (segunda ocasião), dado a classe i no tempo $t$ (primeira ocasião); $N j=$ o número total de árvores no estado $i$ no tempo $t$.

Para fazer a predição após $n$ estações de crescimento a matriz de probabilidade de transição foi elevada a potência $n$ (Equação 3).

$$
p^{(n)}=p i j^{(n)}
$$

Segundo Sanqueta et al. (1996), a estrutura diamétrica da floresta em um tempo futuro é obtida pela equação (4).

$$
E_{1}=A \times E_{0}+R
$$

em que: $E_{l}=$ Estoque no momento um, no tempo de predição futuro; $A=$ é a matriz de probabilidade de transição das árvores nas classes diamétricas; $E_{0}=$ Estoque no momento zero, ou seja, a distribuição diamétrica antes da predição; $R=$ recrutamento. 
A prognose foi feita por grupo ecológico, para tanto as espécies foram classificadas em três grupos: espécies tolerantes à sombra, espécies intolerantes à sombra e intermediárias, como proposto por Jardim et al. (1996) e classificadas em Oliveira et al. (2017).

Foi realizada a prognose de 17 espécies comerciais exploradas em 1997 (Tabela 1). Os indivíduos de Caryocar villosum (piquiá), Couratari oblongifolia (tauarí), Dimorphandra gardneriana (fava-mapuxiqui), Dinizia excelsa (angelim-pedra), Hymenaea sp (angelim-vermelho), Manilkara bidentata (maparajuba), Parkia pendula (fava-bolota) e Pseudopiptadenia suaveolens (faveira-folha-fina) não estavam nas parcelas amostrais da população adulta, portanto, não há dados para análise. A ausência dessas árvores não afetou o estudo, pois sem essas espécies o volume planejado é de $24,86 \mathrm{~m}^{3} \mathrm{ha}^{-1}$, maior que o volume real extraído $\left(23 \mathrm{~m}^{3} \mathrm{ha}^{-1}\right)$. Com isso, para análise dos resultados da pesquisa, considera-se o volume explorado igual a $24,86 \mathrm{~m}^{3} \mathrm{ha}^{-1}$.

Após estimar o número de indivíduos por hectare pelo modelo matriz de transição, foi calculado o volume de madeira pela equação de simples entrada, específica para a área de estudo, proposta por Baima et al. (2001) para árvores em pé com DAP $\geq 20 \mathrm{~cm}(\mathrm{R} 2=$ 0,9391; índice de Furnival = 0,9093; e CV=21,8\%) (Equação 5).

$$
\ln V=-7,49337+2,086952 \ln (D)
$$

em que $V=$ Volume comercial com casca; $D=$ Diâmetro a altura do Peito (DAP) de cada árvore; $L n=$ logaritmo natural.

Com isso, foi definido o ciclo de corte ideal para o grupo de espécies exploradas, considerando que, de acordo com a legislação ambiental, o ciclo definido é de 27 anos (2024), para essa área (Equação 6).

$$
C C=V E / 0,86
$$

em que: $C C=$ Ciclo de corte; $V E=$ volume explorado em $1997\left(23 \mathrm{~m}^{3} \mathrm{ha}^{-1}\right) ; 0,86 \mathrm{~m}^{3} / \mathrm{ha} / \mathrm{ano}$ $=$ produtividade anual da floresta manejada para o grupo de espécies comerciais, quando não houver estudos para a área, para PMFS com uso de máquinas para arraste de toras (BRASIL, 2006).

\section{Resultados e discussão}

\subsection{Prognose da distribuição diamétrica}

Das 17 espécies comerciais exploradas presentes na área amostral, 5 foram classificadas como tolerantes à sombra (T. rhoifolia, S. rubra, A. giganteum, M. paraenses and $Z$. racemosa), 5 intolerantes à sombra ( $H$. courbaril, $R$. albiflora, A. jupunba, $T$. amazonia and $H$. excelsum) e 7 intermediárias (B. acutifolium subsp. Obovatum, $C$. glabrum, G. glabra, M. elata, P. gigantocarpa, S. excelsa and V. americana).

Fez-se a prognose da distribuição diamétrica por grupos ecológicos partindo do ano de 2007 até 2028. As matrizes de probabilidade de transições elaboradas no período 2007- 
2010 para as espécies tolerantes, intolerantes e intermediárias podem ser observadas na tabela 2,3 e 4 , respectivamente.

Tabela 2 - Matriz de probabilidade de transição com intervalo de 3 anos para as espécies tolerantes

\begin{tabular}{|c|c|c|c|c|c|c|c|}
\hline \multirow{2}{*}{$\begin{array}{c}\text { Classe Diamétrica } \\
\text { (2010) }\end{array}$} & \multicolumn{7}{|c|}{ Classe Diamétrica (2007) } \\
\hline & $5-15$ & $15-25$ & $25-35$ & $35-45$ & $45-55$ & $55-65$ & $>65$ \\
\hline $5-15$ & 0,71 & & & & & & \\
\hline $15-25$ & 0,10 & 1,00 & & & & & \\
\hline $25-35$ & & 0,00 & 0,56 & & & & \\
\hline $35-45$ & & & 0,44 & 1,00 & & & \\
\hline $45-55$ & & & & 0,00 & 1,00 & & \\
\hline $55-65$ & & & & & 0,00 & 0,25 & \\
\hline$>65$ & & & & & & 0,00 & 1,00 \\
\hline & abilid & árvore & permane & erem na & nesma cla & se de $\mathrm{D}$ & \\
\hline & babilid & vore & migrare & para a & isse de $\mathrm{D}$ & P segui & \\
\hline
\end{tabular}

Tabela 3 - Matriz de probabilidade de transição com intervalo de 3 anos para as espécies intolerantes

\begin{tabular}{|c|c|c|c|c|c|c|c|}
\hline \multirow{2}{*}{$\begin{array}{c}\text { Classe Diamétrica } \\
(\mathbf{2 0 1 0})\end{array}$} & \multicolumn{7}{|c|}{ Classe Diamétrica (2007) } \\
\hline & $5-15$ & $15-25$ & $25-35$ & $35-45$ & $45-55$ & $55-65$ & $>65$ \\
\hline $5-15$ & 0,56 & & & & & & \\
\hline $15-25$ & 0,19 & 0,00 & & & & & \\
\hline $25-35$ & & 0,00 & 1,00 & & & & \\
\hline $35-45$ & & & 0,00 & 1,00 & & & \\
\hline $45-55$ & & & & 0,00 & 1,00 & & \\
\hline $55-65$ & & & & & 0,00 & 1,00 & \\
\hline$>65$ & & & & & & 0,00 & 1,00 \\
\hline & $\begin{array}{l}\text { abil } \\
\text { abil }\end{array}$ & $\begin{array}{l}\text { árvc } \\
\text { árvo }\end{array}$ & $\begin{array}{l}\text { erma } \\
\text { nigra }\end{array}$ & $\begin{array}{l}\text { erem } n \\
\text { para a }\end{array}$ & $\begin{array}{l}\text { mesma } \\
\text { asse d }\end{array}$ & $\begin{array}{l}\text { asse de } D \\
\text { AP segui }\end{array}$ & \\
\hline
\end{tabular}


Tabela 4 - Matriz de probabilidade de transição com intervalo de 3 anos para as espécies intermediárias

\begin{tabular}{|c|c|c|c|c|c|c|c|}
\hline \multirow{2}{*}{$\begin{array}{c}\text { Classe Diamétrica } \\
\text { (2010) }\end{array}$} & \multicolumn{7}{|c|}{ Classe Diamétrica (2007) } \\
\hline & $5-15$ & $15-25$ & $25-35$ & $35-45$ & $45-55$ & $55-65$ & $>65$ \\
\hline $5-15$ & 0,60 & & & & & & \\
\hline $15-25$ & 0,024 & 0,51 & & & & & \\
\hline 25-35 & & 0,00 & 0,83 & & & & \\
\hline $35-45$ & & & 0,16 & 0,69 & & & \\
\hline $45-55$ & & & & 0,00 & 1,00 & & \\
\hline $55-65$ & & & & & 0,00 & 0,25 & \\
\hline$>65$ & & & & & & 0,75 & 1,00 \\
\hline
\end{tabular}

Pode-se verificar a ausência de indivíduos na classe 15-25 para intolerantes à sombra (Tabela 3). De acordo com Scolforo et al. (1998), isso significa probabilidade zero de transição diamétrica, ocorrendo o estado absorvente. Isso não aponta que a floresta não esteja em seu processo dinâmico. Segundo Lisboa (2014), mesmo neste estado, a mortalidade e o recrutamento continuam a ocorrer sem que, no entanto, a floresta sofra mudanças drásticas em sua estrutura. Esse fato também foi relatado por Stepka et al. (2010).

Observou-se que, para os três grupos ecológicos, a maioria das árvores retrataram maior probabilidade de permanecerem na mesma classe diamétrica, pois não apresentaram um expressivo crescimento em diâmetro para mudar de classe.

Costa (2000) avaliou o crescimento de um povoamento na Floresta Nacional do Tapajós e observou que, em 16 anos, o crescimento médio em diâmetro foi de $0,30 \mathrm{~cm}_{\text {ano- }}^{-}$ ${ }^{1}$ para todas as espécies e $0,35 \mathrm{~cm}^{2} \mathrm{ano}^{-1}$ para espécies comerciais com DAP $\geq 5 \mathrm{~cm}$. Nessa mesma área, em um período de 8 anos após a exploração, Carvalho et al., (2004) verificaram um crescimento de $0,37 \mathrm{~cm}^{2} \mathrm{ano}^{-1}$ para as árvores localizadas nas parcelas permanentes em que foram explorados indivíduos com DAP $\geq 45 \mathrm{~cm}$ e $0,36 \mathrm{~cm}^{-1} \mathrm{ano}^{-1}$ na área para os indivíduos colhidos com DAP $\geq 55 \mathrm{~cm}$. Silva (1998), em seu estudo no sudeste paraense, constatou que as árvores que receberam iluminação total cresceram em média $0,55 \mathrm{~cm}^{2} \mathrm{no}^{-}$ 1 , as árvores parcialmente sombreadas cresceram $0,31 \mathrm{~cm} \mathrm{ano}^{-1} \mathrm{e}$ aquelas que não receberam iluminação cresceram, apenas, $0,22 \mathrm{~cm}^{2} \mathrm{ano}^{-1}$.

Ressalta-se que o período inicial para a prognose foi de 13 anos após a exploração (2007-2010), portanto, as clareiras maiores, provenientes da exploração, já se encontram fechadas, existindo apenas as clareiras naturais, e, com isso, pouco iluminação até o piso florestal, reiterando o lento crescimento e justificando a frequência zero na transição da maioria das classes. Silva et al. (1999) concluíram que o estímulo resultante da abertura do dossel pela extração de árvores é passageiro, visto que nos primeiros anos de observação o 
crescimento das árvores e o aumento do número de ingresso ocorreram intensamente depois entraram em declínio.

Após determinar a matriz de probabilidade (tabelas 2,3 e 4 ), realizou-se a prognose da estrutura diamétrica para os anos 2013, 2016, 2019, 2022, 2025 e 2028 (Tabela 5).

Tabela 5 - Indivíduos por hectare projetados para os anos 2013, 2016, 2019, 2022, 2025 e 2028, por classe diamétrica, para os grupos ecológicos tolerante à sombra, intolerante à sombra e intermediária

\begin{tabular}{|c|c|c|c|c|c|c|c|}
\hline $\begin{array}{c}\text { Grupo } \\
\text { ecológico }\end{array}$ & C.D. & $\begin{array}{l}\text { P. } 2013 \\
\left(\mathrm{~N} \mathrm{ha}^{-1}\right)\end{array}$ & $\begin{array}{l}\text { P. } 2016 \\
\left(\mathrm{~N} \mathrm{ha}^{-1}\right)\end{array}$ & $\begin{array}{l}\text { P. } 2019 \\
\left(\mathrm{~N} \text { ha }^{-1}\right)\end{array}$ & $\begin{array}{l}\text { P. } 2022 \\
\left(\mathrm{~N} \text { ha }^{-1}\right)\end{array}$ & $\begin{array}{l}\text { P. } 2025 \\
\left(\mathrm{~N} \mathrm{ha}^{-1}\right)\end{array}$ & $\begin{array}{l}\text { P. } 2028 \\
\left(\mathrm{~N} \mathrm{ha}^{-1}\right)\end{array}$ \\
\hline \multirow{7}{*}{$\begin{array}{l}\text { Tolerante à } \\
\text { sombra }\end{array}$} & $5-15$ & 8,0409 & 6,2577 & 4,9932 & 4,0964 & 3,4605 & 3,0095 \\
\hline & $15-25$ & 2,6901 & 3,4697 & 4,0764 & 4,5605 & 4,9576 & 5,2931 \\
\hline & $25-35$ & 1,2500 & 0,7031 & 0,3955 & 0,2225 & 0,1251 & 0,0704 \\
\hline & $35-45$ & 2,6389 & 3,1858 & 3,4934 & 3,6664 & 3,7637 & 3,8185 \\
\hline & $45-55$ & 0,5556 & 0,5556 & 0,5556 & 0,5556 & 0,5556 & 0,5556 \\
\hline & $55-65$ & 0,2778 & 0,0694 & 0,0174 & 0,0043 & 0,0011 & 0,0003 \\
\hline & $>65$ & 1,6667 & 1,6667 & 1,6667 & 1,6667 & 1,6667 & 1,6667 \\
\hline \multirow{7}{*}{$\begin{array}{c}\text { Intolerante à } \\
\text { sombra }\end{array}$} & $5-15$ & 1,8056 & 1,5712 & 1,4393 & 1,3652 & 1,3235 & 1,3000 \\
\hline & $15-25$ & 0,4167 & 0,3385 & 0,2946 & 0,2699 & 0,2560 & 0,2482 \\
\hline & $25-35$ & 0,5556 & 0,5556 & 0,5556 & 0,5556 & 0,5556 & 0,5556 \\
\hline & $35-45$ & 0,5556 & 0,5556 & 0,5556 & 0,5556 & 0,5556 & 0,5556 \\
\hline & $45-55$ & 0,5556 & 0,5556 & 0,5556 & 0,5556 & 0,5556 & 0,5556 \\
\hline & $55-65$ & 0,5556 & 0,5556 & 0,5556 & 0,5556 & 0,5556 & 0,5556 \\
\hline & $>65$ & 1,6667 & 1,6667 & 1,6667 & 1,6667 & 1,6667 & 1,6667 \\
\hline \multirow{7}{*}{ Intermediária } & $5-15$ & 21,7563 & 14,1513 & 9,5931 & 6,8610 & 5,2234 & 4,2419 \\
\hline & $15-25$ & 4,7952 & 2,9688 & 1,8545 & 1,1765 & 0,7649 & 0,5157 \\
\hline & $25-35$ & 5,0505 & 4,1740 & 3,4496 & 2,8509 & 2,3561 & 1,9472 \\
\hline & $35-45$ & 3,2828 & 3,0798 & 2,7999 & 2,4908 & 2,1813 & 1,8880 \\
\hline & $45-55$ & 5,0000 & 5,0000 & 5,0000 & 5,0000 & 5,0000 & 5,0000 \\
\hline & $55-65$ & 0,8333 & 0,2083 & 0,0521 & 0,0130 & 0,0033 & 0,0008 \\
\hline & $>65$ & 4,7222 & 5,3472 & 5,5035 & 5,5425 & 5,5523 & 5,5547 \\
\hline
\end{tabular}




\subsection{Volume e Ciclo de Corte}

O diâmetro mínimo das árvores comerciais colhidas era de $65 \mathrm{~cm}$, portanto, fez-se o cálculo do volume para os indivíduos a partir desse DAP. Aplicando a equação de volume proposta por Baima et al. (2001), verifica-se os resultados na Tabela 6.

Tabela 6 - Volume estimado para os anos 2013, 2016, 2019, 20222025 e 2028 para espécies exploradas divididas em grupos ecológicos e para todas as espécies em uma floresta tropical de terra firme na Amazônia Brasileira

\begin{tabular}{cccccccc}
\hline \multirow{2}{*}{$\begin{array}{c}\text { Grupo } \\
\text { Ecológico }\end{array}$} & $\begin{array}{c}\text { Volume } \\
\text { explorado } \\
(\mathbf{1 9 9 7 )}\end{array}$ & \multicolumn{5}{c}{ Volume estimado para as árvores com DAP $>\mathbf{6 5} \mathbf{~ c m}$} \\
\cline { 3 - 8 }$\left(\mathbf{m}^{\mathbf{3}} \mathbf{h a}^{-1}\right)$ \\
& & $\mathbf{2 0 1 3}$ & $\mathbf{2 0 1 6}$ & $\mathbf{2 0 1 9}$ & $\mathbf{2 0 2 2}$ & $\mathbf{2 0 2 5}$ & $\mathbf{2 0 2 8}$ \\
\hline $\begin{array}{c}\text { Tolerante à } \\
\text { sombra }\end{array}$ & 3,21 & 5,636 & 5,636 & 5,636 & 5,636 & 5,636 & 5,636 \\
\hline $\begin{array}{c}\text { Intolerante à } \\
\text { sombra }\end{array}$ & 3,4 & 5,636 & 5,636 & 5,636 & 5,636 & 5,636 & 5,636 \\
\hline Intermediária & 18,25 & 15,969 & 18,083 & 18,611 & 18,743 & 18,776 & 18,784 \\
\hline $\begin{array}{c}\text { Todas as } \\
\text { espécies }\end{array}$ & 24,86 & 27,241 & 29,355 & 29,883 & 30,015 & 30,048 & 30,057 \\
\hline
\end{tabular}

Conforme observa-se na tabela 6 , as espécies tolerantes e intolerantes à sombra atingiram o volume explorado em 2013, 16 anos após a exploração. Já as espécies intermediárias foi no ano de 2019, 22 anos depois da colheita.

Resultados semelhantes foram encontrados por outros pesquisadores. Reis et al. (2017), ao estimar o ciclo de corte para a mesma área de estudo utilizando o Método Mexicano de Ordenamento Florestal (MMOF), recomendou o ciclo de corte de 22 anos, considerando apenas a recuperação do volume retirado, que é o principal objetivo do manejo florestal. Souza et al. (2004), indicam a intensidade de corte de 25 a $35 \mathrm{~m}^{3} \mathrm{ha}^{-1}$, com ciclos de corte de 17 e 18 anos, pois proporciona a utilização do recurso sem comprometer a sua capacidade de renovação ou sustentação da floresta manejada. Bom (1996), constatou um ciclo de corte de 23 anos com uma intensidade de $22,53 \mathrm{~m}^{3} \mathrm{ha}^{-1}$.

Schneider e Finger (2000) afirmam que é necessário que a floresta tropical possa repor o volume de corte, durante o período de tempo definido pelo ciclo de corte estabelecido, garantindo a sustentabilidade de produção da floresta. Esse tempo não necessariamente coincide com o estipulado na legislação florestal, de 25 a 35 anos, como foi ratificado pelos resultados da presente pesquisa e autores supracitados.

As espécies tolerantes e intolerantes à sombra apresentaram o mesmo volume para todos os anos. O mesmo crescimento das tolerantes e intolerantes ocorre pelo fato de que mesmo tolerando o sombreamento, estas espécies se beneficiam da abertura do dossel 
acelerando o seu crescimento, considerando esse comportamento como oportunista, em termos de crescimento, devido à melhoria nas condições de luz (Costa et al., 2007), ratificando que o grupo das espécies tolerantes à sombra possui grande elasticidade ambiental, se adaptando a uma grande amplitude de ambientes (JARDIM et al., 2007; CHAGAS et al., 2012).

Costa et al. (2007) afirmam que apesar de determinadas espécies serem tolerantes à sombra, estas se beneficiam com a abertura do dossel para crescerem. Korning e Balslev (1994), avaliando espécies arbóreas em floresta tropical na Amazônia, identificaram cinco espécies preferenciais de dossel ou subdossel, tolerantes à sombra, mas que respondiam, em termos de crescimento, as melhorias nas condições luminosas. Isso corrobora o comentário de Costa et al. (2008) de que independente das características intrínsecas das espécies (grupo ecológico, fatores genéticos etc.), a entrada de luz na copa das árvores estimula o crescimento de todas as espécies.

As espécies comerciais, muitas das quais são secundárias tardias ou oportunistas e intolerantes à sombra, terão sua capacidade de recobrimento reduzido pela remoção de matrizes e manutenção dos indivíduos de espécies não comerciais. Todavia, com a exploração, isso pode ser compensado pela criação de ambientes mais favoráveis para o recrutamento e crescimento das espécies mais intolerantes (Jardim, 2015).

As espécies intermediárias apresentaram um ciclo de corte maior em relação aos outros grupos, devido, provavelmente, a demora na recuperação do volume ocasionado pelo maior número de indivíduos com DAP maior que $65 \mathrm{~cm}$, maior taxa de mortalidade e ingresso. De acordo com Jardim (2015), para esse grupo regenerar e crescer depende de luz em graus variados, que são disponíveis em clareiras de tamanhos médio a pequeno. A exploração florestal, que proporciona maiores clareiras, pode ter inibido o crescimento dessas espécies.

O resultado do Ciclo de Corte para grupos ecológicos de espécies exploradas demonstra que o tempo para outra exploração na mesma área estabelecido pela legislação ambiental Brasileira, IN 5 de 2006, independente da espécie que está sendo manejada, pode não ser sustentável por tratar de uma forma igual espécies com ritmos de crescimento diferentes (BRAZ et al., 2012).

\section{Conclusões}

Verificou-se que as espécies tolerantes e intolerantes recuperaram o estoque colhido em um período de 16 anos e as espécies intermediárias apresentaram um ciclo de corte de 22 anos, indo de encontro ao tempo estipulado em lei, de 27 anos.

A exploração florestal necessita que as espécies sejam classificadas conforme suas demandas ecofisiológicas e se estabeleça um ciclo de corte compatível com a recuperação do volume extraído de cada grupo, garantindo a sustentabilidade ambiental e financeira da atividade. 


\section{Agradecimentos}

Aos revisores e editores pelos comentários e sugestões e ao CNPq pelo apoio financeiro do projeto "Avaliação da dinâmica florestal após exploração madeireira seletiva”, edital Universal 2006, Processo 470769/2006-8.

OLIVEIRA, L. C. L. Q; JARDIM, F. C. F.; GOMES, J. M. Prediction of the commercial tree species cutting cycle by ecological groups in a forest in the Brazilian Amazon. Rev. Bras. Biom. Lavras, v.38, n. 1, p.18-34, 2020.

- ABSTRACT: This research aimed to prognosis the diameter distribution of the forest using the Transition Matrix model for species classified as tolerant, intolerant and intermediate and to define the cutting cycle by ecological groups, in order to verify if the time defined in the Brazilian environmental legislation is compatible with the recovery and growth of tree species. The research area is located in Moju, Pará, Brazil. Nine clearings were selected, around which 4 sample plots of $500 \mathrm{~m}^{2}$ were installed. All individuals with $\mathrm{DBH} \geq 5 \mathrm{~cm}$ were measured. For the prognosis, actual data of the years 2007 and 2010 were used and the projections were made for a 3 years period. With predicted data, the volume estimation was performed up to 31 years after the lumbering. It was found that for the tolerant and intolerant species, the ideal cutting cycle is 16 years, and for the intermediate ones, 22 years.

- KEYWORDS: Selective logging; forest management; transition matrix; diametric distribution.

\section{Referências}

APG III. An update of the angiosperm phylogeny group classification for the orders and families of flowering plants: APG III. Botanical Journal of the Linnean Society, v.161, p.105- 121, 2009.

AVERY, T. E.; BURKHART, H. E. Forest measurements. New York: McGraw-Hill, 1994. 432p.

BAIMA, A. M. V.; SILVA, S. M. A.; SILVA, J. N. M. Equações de volume para floresta tropical de terra firme em Moju, PA. In: SILVA, J. N. M.; CARVALHO, J. O. P.; YARED, J. A C. (Eds.). A silvicultura na Amazônia Oriental: contribuições do projeto Embrapa/DFID. Belém: Embrapa Amazônia Oriental - DFID, 2001. p. 367-392.

BAKER, F. S. A revised tolerance table. Journal of Forestry, v.47, p.179-181, 1949.

BOM, R. P. Proposição de um sistema de manejo para floresta nativa objetivando a sustentabilidade da produção. 1996. 216 f. Tese (Doutorado em Ciências Florestais). Universidade Federal do Paraná, Paraná, 1996.

BRASIL. MINISTÉRIO DO MEIO AMBIENTE. INSTRUÇÃO NORMATIVA N ${ }^{\circ}$ 5, DE 11 DE DEZEMBRO DE 2006. Dispõe sobre procedimentos técnicos para elaboração, apresentação, execução e avaliação técnica de Planos de Manejo Florestal SustentávelPMFSs nas florestas primitivas e suas formas de sucessão na Amazônia Legal, e dá outras providências. Diário Oficial da República Federativa do Brasil, Brasília, DF, 5 mar. 2006. 
BRASIL, CONSELHO NACIONAL DO MEIO AMBIENTE. RESOLUÇÃO N 406, DE 02 DE FEVEREIRO DE 2009. Estabelece parâmetros técnicos a serem adotados na elaboração, apresentação, avaliação técnica e execução de Plano de Manejo Florestal Sustentável PMFS com fins madeireiros, para florestas nativas e suas formas de sucessão no bioma Amazônia. Diário Oficial da República Federativa do Brasil, n 26, 06 de fev. 2009.

BRAZ, E. M.; SCHNEIDER, P. R.; MATTOS, P. P.; SELLE, G.L.; THAINES, F.; RIBAS, L. A.; VUADEN, E. Taxa de corte sustentável para manejo das florestas tropicais. Ciência Florestal, v.22, n.1, p.137-145, 2012.

BUDOWSKI, G. Distribución de las especies del bosque lluvioso tropical americano a la luz de los procesos sucesionales. Turrialba, v.15, n.1, p.40-42, 1965.

CARVALHO, J. O. P.; SILVA, J. N. M.; LOPES, J. C. A. Growth rate of a terra firme rain forest in brazilian amazonia over an eight-years period in response to logging. Acta Amazonica, v.34, n.2, p.209-217, 2004.

CHAGAS, R. S.; GOMES, J. M. G.; CARVAlHO, J. O. P. de; FERREIRA, J. E. R. Sobrevivência e crescimento de plântulas de Manilkara huberi Chevalier durante cinco anos em clareiras causadas pela exploração de impacto reduzido na Amazônia brasileira. Scientia Forestalis, v.40, n.95, p.417-424, 2012.

COELHO, R. F. R.; MIRANDA, I. S.; MITJA, D. Caracterização do processo sucessional no Projeto de Assentamento Benfica, sudeste do estado do Pará, Amazônia oriental. Boletim Museu Paraense Emílio Goeldi, Ciências Naturais, v.7, n.3, p.251-282, 2012.

CORNELISSEN, J. H. C.; LAVOREL, S.; GARNIER, E.; DÍAZ, S.; BUCHMANN, N.; GURVICH, D. E.; REICH, P. B.; STEEGE, H. ter; MORGAN, H. D.; HEIJDEN, M. G. A. van der; PAUSAS, J. G.; POORTER, H. A handbook of protocols for standardised and easy measurement of plant functional traits worldwide. Australian Journal of Botany, v.51, p.335-380, 2003.

COSTA, D. H. M. Dinâmica da composição florística e crescimento de uma área de floresta de terra-firme na FLONA do Tapajós, durante 18 após a colheita de madeiras. 2000. 120f. Dissertação (Mestrado em Ciências Florestais), Universidade Federal Rural da Amazônia, Pará, 2000.

COSTA, D. H. M.; CARVALHO, J. O. P.; BERG, E. V. D. Crescimento diamétrico de maçaranduba (Manilkara huberi Chevalier) após a colheita da madeira. Amazônia: Ciência \& Desenvolvimento, v.3, n.5, p.65-76, 2007.

COSTA, D. H. M.; SILVA, J. N. M.; CARVALHO, J. O. P. Crescimento de árvores em uma área de terra firme na floresta nacional do tapajós após a colheita de madeira. Revista de Ciências Agrárias, v.50, p.63-76, 2008.

DAVIS, K. P. Forest management: regulation and valuation. New York: McGraw-Hill, 1966. 519p.

DAVIS, L. S.; JOHNSON, K. N. Forest management. 3.ed. New York: McGraw-Hill, 1987. 790p.

DENSLOW, J. S. Gap partioning among tropical rain forest trees. Biotropica, v.12, p.47$55,1980$. 
DUAH-GYAMFI, A.; SWAINE, E. K.; ADAM, K. A.; PINARD, M. A.; SWAINE, M. D. Can harvesting for timber in tropical forest enhance timber tree regeneration? Forest ecology and Management, v.314, p.26-37, 2014.

HARTSHORN, G. S. Neotropical forests dynamics. Biotropica, v.12, p.23-30, 1980.

HUMBERT, L.; GAGNON, D.; KNEESHAW, D.; MESSIER, C. A shade tolerance index for common understory species of northeastern North America. Ecological Indicators, v.7, p.195-207, 2007.

JARDIM, F. C. S.; SOUZA, A. L.; BARROS, N. F.; SILVA, E.; MACHADO, C. C.; SILVA, A. F. Agrupamento das espécies arbóreas de uma floresta equatorial na região de Manaus-AM. Boletim da Fcap, v.26, p.7-29, 1996.

JARDIM, F. C. S.; SERRÃO, D. R.; NEMER, T. C. Efeito de diferentes tamanhos de clareiras, sobre o crescimento e a mortalidade de espécies arbóreas, em Moju-PA. Acta Amazonica, v.37, n.1, p.37-48, 2007.

KAGEYAMA, P. Y.; GANDARA, F. B. Recuperação de áreas ciliares. In: RODRIGUES, R. R.; LOURENÇO FILHO, H. F. (Ed.). Matas ciliares: conservação e recuperação. São Paulo: USP/FAPESP, p.249-270, 2004.

KORNING, J.; BALSLEV, H. Growth rates and mortality patterns of tropical lowland tree species and the relation to forest structure in Amazonian Ecuador. Journal of Tropical Ecology, v.10, p.151-166, 1994.

LISBOA, G. S. Projeção diamétrica em remanescente de floresta ombrófila mista na flona de Irati, PR. 2014. 90f. Monografia (especialização em estatística). Universidade Federal de Santa Maria, Rio Grande do Sul, 2014.

LOPES, J. C. A.; WHITMORE, T. C.; BROWN, N. D.; JENNEING, S. B. Efeito da exploração florestal nas populações de mudas em uma floresta tropical úmida no município de Moju, PA. In: Silva, J. N. M.; Carvalho, J. O. P.; Yared, J. A. G. (Ed.). A silvicultura na Amazônia Oriental: contribuições do projeto Embrapa/DFID. Belém: Embrapa Amazônia Oriental - DFID, 2001. p.227-251.

NASCIMENTO, R. G. M.; MACHADO, S. A.; FIGUEIREDO FILHO, A.; HIGUCHI, N. A growth and yield projection system for a tropical rainforest in the Central Amazon, Brazil. Forest Ecology and Management, v.327, p.201-208, 2014.

OLIVEIRA, L. C. L. Q.; JARDIM, F. C. S.; GOMES, J. M.; RAMOS, E. M. L. S. Classificação ecológica de espécies arbóreas por meio da análise da distribuição diamétrica. Espacios, v.38, n.42, 2017.

REIS, L. P.; SOUZA, A. L.; REIS, P. C. M.; FREITAS, L. J. M.; SILVA, L. F.; REGO, L. J. S. Aplicação do método mexicano de ordenamento florestal (MMOF) para estimar o ciclo e intensidade de corte em Floresta na Amazônia Oriental. Scientia Forestalis, v.45, n.113, p.187-195, 2017.

REFLORA, Programa Reflora. Disponível em: http://reflora.jbrj.gov.br/reflora/, 2017.

SANQUETTA, C. R.; BRENA, D. A.; ANGELO, H.; MENDES, J. B. Matriz de transição para simulação da dinâmica de florestas naturais sob diferentes intensidades de corte. Ciência Florestal, v.6, n.1, p.65-78. 1996. 
SCHNEIDER, P. R.; FINGER, C. A. G. Manejo Sustentado de Florestas Inequiâneas Heterogêneas. Santa Maria: Universidade Federal de Santa Maria. Departamento de Ciências Florestais. 2000. 195 p.

SCOLFORO, J. R. S.; PULZ, F. A.; MELLO, J. M. Modelagem da produção, idade da floresta nativa, distribuição especial das espécies e a análise estrutural. In: SCOLFORO, J, R. S. Manejo florestal. Lavras: UFLA/FAEPE, 1997, p.189-245.

SILVA, E. J. V. Impactos da exploração madeireira predatória e planejada sobre o crescimento e diversidade de espécies arbóreas na Amazonia Oriental. 1998. 82f. Dissertação (Mestrado em Ciências Florestais), ESALQ, Piracicaba, 1998.

SILVA, J. N. M.; CARVALHO, J. O. P.; LOPES, J. DO C. A.; ALMEIDA, B. F.; COSTA, D. H. M.; OLIVEIRA, L. C.; VANCLAY, J. K.; SKOVSGAARD, J. P. Crescimento e produção de uma floresta tropical da Amazônia brasileira treze anos após a exploração. In: SIMPÓSIO SILVICULTURA NA AMAZÔNIA ORIENTAL: Contribuições do Projeto EMBRAPA, 1999, Belém, Anais. Belém: EMBRAPA, p.186-189, 1999.

SILVA, S. M. A. S.; SILVA, J. N. M.; BAIMA, A. M. V.; LOBATO, N. M.; THOMPSON, I. S.; COSTA FILHO, P. P. Impacto da exploração madeireira em floresta de terra firme no município de Moju, estado do Pará. In: SILVA, J. N. M; CARVALHO, J. O. P.; YARED, J. A. G. (Eds.). A silvicultura na Amazônia Oriental: contribuições do projeto Embrapa/DFID. Belém: Embrapa Amazônia Oriental - DFID, 2001. p. 227-251.

SILVA, R. P.; SANTOS, J.; TRIBUZY, E. S.; CHAMBERS, J. Q.; NAKAMURA, S.; HIGUCHI, N. Diameter increment and growth patterns for individual tree growing in Central Amazon, Brazil. Forest Ecology and Management, v.166, p.295-301, 2002.

SOARES, A. A. V.; BOTELHO, S. A.; DAVIDE, A. C.; FARIA, J. M. R. Influence of ecological group composition, plantation spacing and arrangement in the restoration of riparian forest on reservoir shores. Ciência Florestal, v.26, n.4, p.1107-1118, 2016.

SOUZA, D. R.; SOUZA, A. L.; SILVA, M. L.; RODRIGUES, F. L. Ciclo de Corte econômico ótimo em floresta ombrófila densa de terra firme sob manejo florestal sustentável, Amazônia Oriental. Revista Árvore, v.28, n.5, p. 681-689, 2004.

SOUZA, A. L.; SOARES, C. P. B. Florestas nativas: estrutura, dinâmica e manejo. Viçosa: UFV, 2013. 322p.

STEPKA, T. F.; DIAS, A. N.; FIGUEIREDO FILHO, A.; MACHADO, S. A.; SAWCZUK, R. Prognose da estrutura diamétrica de uma Floresta Ombrófila Mista com os métodos razão de movimentos e matriz de transição. Pesquisa Florestal Brasileira, v.30, n.64, p.327-335, 2010.

TEIXEIRA, L. M.; JEFFREY, Q.; CHAMBERS, J. Q.; SILVA, A. R. E; LIMA, A. J. N.; CARNEIRO, V. M. C.; SANTOS, J. dos; HIGUCHI, N. Projeção da dinâmica da floresta natural de Terra-firme, região de Manaus - AM, com o uso da cadeia de transição probabilística de Markov. Acta Amazonica, v.37, n.3, p 377-384, 2007.

VANCLAY, J.K. Modelling forest growth and yield: applications to mixed tropical forests. CAB International, Department of Economics and Natural Resource, Royal Veterinary and Agricultural University, Copenhagen, Denmark, Wallingford, UK, 1994. 312 p. 
WHITMORE, T. C. Tropical rainforest of the Far East. 2.ed. Oxford: Oxford University Press, 1984.

Recebido em 26.10.2018

Aprovado após revisão em 18.11.2019 